Computers \& Operations Research

Special Issue: Operations research for health care delivery

\title{
A multilevel integrative approach to hospital case mix and capacity planning
}

\author{
Guoxuan Ma \& Erik Demeulemeester* \\ Katholieke Universiteit Leuven, Faculty of Business and Economics \\ Department of Decision Sciences and Information Management \\ Naamsestraat 69, B-3000 Leuven, Belgium \\ Tel: +32-16-32.69.72 \\ Fax: +32-16-32.66.24 \\ guoxuan.ma@econ.kuleuven.be \\ erik.demeulemeester@econ.kuleuven.be
}

* Corresponding author 


\title{
A multilevel integrative approach to hospital case mix and capacity planning
}

\begin{abstract}
:
Hospital case mix and capacity planning involves the decision making both on patient volumes that can be taken care of at a hospital and on resource requirements and capacity management. In this research, to advance both the hospital resource efficiency and the health care service level, a multilevel integrative approach to the planning problem is proposed on the basis of mathematical programming modeling and simulation analysis. It consists of three stages, namely the case mix planning phase, the master surgery scheduling phase and the operational performance evaluation phase. At the case mix planning phase, a hospital is assumed to choose the optimal patient mix and volume that can bring the maximum overall financial contribution under the given resource capacity. Then, in order to improve the patient service level potentially, the total expected bed shortage due to the variable length of stay of patients is minimized through reallocating the bed capacity and building balanced master surgery schedules at the master surgery scheduling phase. After that, the performance evaluation is carried out at the operational stage through simulation analysis, and a few effective operational policies are suggested and analyzed to enhance the trade-offs between resource efficiency and service level. The three stages are interacting and are combined in an iterative way to make sound decisions both on the patient case mix and on the resource allocation.
\end{abstract}

Keywords: health care, case mix and capacity planning, master surgery schedule, multilevel, resource efficiency, service level 


\section{$1 \quad$ Introduction}

In Belgium, the total health expenditures increase year after year and may expand potentially in the future. Actually, 15.38 billion Euro was spent in the health care sector in 2003 , whereas this number was increased to 20.70 billion Euro in 2008 (RIZIV [1]). This represents an increase from $9.6 \%$ of the gross domestic product in 2003 to $10.2 \%$ in 2008 (OECD [2]). On the one hand, this increment attributes to an aging population growth combined with new and more expensive treatments, but on the other hand it is also the result of the incapability of the hospital to handle the decision making at strategic, tactical and operational levels in an integrative way, resulting in inefficiencies of the hospital operations.

Moreover, an inefficient hospital management will not only bring huge economic pressures to a hospital, but also result in the waste of precious resources and the inefficiency of the patient flow, as exemplified by long waiting lists, surgery cancellations or patient misplacements, which will directly impact the health care service level. Particularly, under the limited resource capacity in a given hospital, the inevitable variability within health systems, both from the hospital supply side (e.g., a machine breakdown, surgeons called away for emergency) and from the patient demand side (e.g., random patient arrivals, stochastic surgery durations), will undoubtedly further add to this inefficiency. Therefore, it becomes important for the health care sector to improve both the hospital resource efficiency and the health service level, and it becomes increasingly necessary for hospitals to investigate the production management problem in a systematic way.

Firstly, in order to mitigate the increasing economic pressures, hospitals are prone to advance the financial contribution of their resources through an efficient capacity allocation and management. Naturally, it is expected that the available resource capacity may match the stochastic patient demand as perfectly as possible and the utilizations of multifold resources (i.e., hospital beds, 
operating rooms (ORs), nursing staff, etc.) may be coordinated as best as possible. Nevertheless, due to the scarcity of resources and the variability (e.g., the random patient arrivals, the variable length of stays (LOSs), etc.) within the patient flow, the capacity management problem seems to be quite complicated. But it is fortunate that, as pointed out by Michael Carter [3], these complex problems within health care systems could benefit from operational research methods, such as mathematical programming, discrete-event simulation, and so on.

To implement target patient throughputs, Vissers [4] combines hospital production management and resource capacity planning together, and proposes a formalized method to allot the resource capacity within a whole hospital. Two mathematical models are built to calculate the resource requirements of each specialty. One supports long-term decisions about the resources required to match the future patient demand, and another supports decision making at the medium-term level for balancing the resource requirements of various types. Similarly, for the financial purpose, it is also beneficial for a hospital to integrate the production management and the capacity planning. Here, a hospital pursues to choose the patient mix and volume of different pathology types that can bring maximum profits and to determine the corresponding hospital-wide resource allocation. The mix and volume of different types of patients are referred to as the patient case mix [5] in this context, and the production management problem is referred to as the hospital case mix and capacity planning problem. Clearly, this hospital planning problem involves the decision making both on patient volumes and on resource requirements and allocation, and thus concerns both the patient volumes planning and control level and the resources planning and control level within the health care production control framework of Vissers et al. [6].

Hospitals are classified into two categories: constrained profit satisfiers and profit maximizers [7], according to the different profit targets of health care providers. Constrained profit satisfiers 
are motivated by professional interests rather than economic returns. A profit satisfier hospital seeks for the preferred case mix pattern or other preset objectives on condition that it can manage to break even without violating the capacity constraints [8]. On the other hand, profit maximizer hospitals are assumed to be positioned in a competitive business environment and thus they are willing to choose the patient cases that will bring the maximum rewards (e.g., [9-11]). In these papers, the mathematical programming models (i.e., linear programming, integer programming, goal programming) are deployed to solve the intended patient case mix. Moreover, in previous research [11], an efficient solution method for huge integer programs, branch-and-price [12], is presented and applied to solve the case mix problem optimally. In addition, to reflect and contain the variability in the system (e.g., the variable LOS of patients) into the planning model, both Utley et al. [13] and Adan et al. [14] successfully translate the stochastic resource requirements into the mathematical modeling by replacing the expected LOS with its probability distribution, and receive a better result from these stochastic models when solving the patient case mix and/or the allocation of resources in real configurations.

Secondly, as mentioned above, the inevitable variability in the health care system will not only influence the resource utilization [15-17], but also affect the service level of health care delivery [18-21]. Specifically, both the unpredictable patient demand (e.g., emergency admissions [15]) and the variable length of stay of patients (e.g., $[13,14,16])$ have a considerable impact on the fluctuation of bed capacity requirements. Moreover, Green [17] points out that the bed capacity planning should not be based on the target occupancy level only, but be connected with measures of patient delays, such as patient refused admission rate [18], patient waiting time [19], surgery cancellation [20], and patient misplacement [21]. To assess the operational performance and to examine the health care service level, a queueing model is adopted in [18] and simulation models 
are applied in [19-21]. In addition, besides the variability, changes in a cyclic master surgery schedule itself also affect bed consumptions. For instance, Beliën et al. [22] present a software system that visualizes the impact of the master surgery schedule on the demand for other various resources throughout the rest of the hospital.

Apparently, the balanced use of various resources will not only increase the resource efficiency but advance the patient service level. Hence, it is significant to harmonize the utilization of, e.g., operating rooms and beds, in order to acquire an efficient patient flow in the health care system. Beliën and Demeulemeester [23] take into account the resulting daily bed occupancy levels when building cyclic master surgery schedules. The variable LOS of patients will bring fluctuations in the bed capacity requirement and deviations of the real bed demand from the assigned capacity, which causes the bed shortage phenomenon. Then, the bed shortage will lower the patient service level, since it either makes the scheduled surgery to be cancelled [20] or it makes the operated patient to be misplaced [21]. Hence, it is meaningful to seek for a balanced cyclic master surgery schedule through minimizing the total expected bed shortages [23]. Besides, van Oostrum et al. [24] also incorporate maximizing OR utilizations and leveling bed requirements together when scheduling the surgery procedures into operating rooms.

In addition, there also exist a number of articles [25-27] applying an integrative approach to the hospital planning problems. Bretthauer and Côté [25] develop an optimization/queueing network model to determine resource requirements, which minimizes the capacity costs while controlling the customer service by enforcing a set of performance constraints. The queueing network model allows to involve the stochastic property of health care systems and to estimate the service levels within an optimization framework. Butler et al. [26] propose a two-phase model-based approach to the hospital layout problem. A quadratic integer goal programming model is built to determine 
a hospital configuration and the allocation of beds to health services in the first phase. Then, the detailed ramification of the suggested layout is assessed in the second phase through a simulation model. Oddoye et al. [27] also combine simulation analysis with goal programming modeling for the healthcare planning in order to achieve an optimal clinical workflow.

In this research, to overcome the increasing economic pressures on hospitals and the inefficiency in patient flows, the case mix and capacity planning problem is studied comprehensively, which on the one hand maximizes the financial contribution of the given resources at a hospital, and on the other hand improves the health care service level under the variability condition. To achieve the efficient trade-offs between resource efficiency and service level, an integrative three-phased solution approach is proposed, in which the first phase aims at maximizing the overall profits, the second phase is focused on reducing the total expected bed shortages, and the third phase works on assessing the operations performance and on analyzing the suggested effective policies. Each phase has its own emphasis and merits, and their solutions complement each other and complete the sound decision making for the hospital planning problem.

The remainder of this paper is structured as follows. Section 2 describes the hospital case mix and capacity planning problem briefly and addresses some concerned matters being valuable to the planning process. Section 3 specifies the proposed solution methodology for the planning problem, which is a multilevel model-based integrative approach on the basis of operational research methods, i.e., mathematical (integer linear) programming and discrete-event simulation. The methodology framework is formulated and explained in detail, and each phase is elaborated respectively. Afterwards, an example is applied to demonstrate the efficiency and the importance of the designed integrative method in Section 4. Finally, in Section 5 we draw the conclusions of this paper and mention some ideas for further developments. 


\section{Problem description}

As discussed above, the hospital case mix and capacity planning problem concerns the decision making both at the patient volumes planning and control level and at the resources planning and control level of the hierarchical framework for production control of hospitals [6]. Both levels consider an entire hospital as the main unit of analysis. The patient volumes planning and control level with a planning horizon of 1 to 2 years concerns the long-term decision making on patient flows and resources. Decisions regarding patient flows consist of the annual number of patients that can be treated per pathology group, namely the patient case mix, while decisions regarding resources consist of the capacity requirement of each specialty within the hospital. The objective is to match patient demands and supplied resources as best as possible. The resources planning and control level with a planning horizon of 3 months to 1 year concerns the decision making at the medium-term level, which consists of the time-phased resource allocation and the capacity coordination requirement. The objective is to maximally coordinate the utilization of multifold resources (e.g., operating rooms and beds) within the whole hospital. Ignoring this coordination may result in capacity losses, i.e., a poor performance in resource utilization. Hence, integrating the decision making at both levels is beneficial to produce an efficient patient flow and thus to advance the hospital's production efficiency.

A hospital might be considered as a production system, in which the scarce resources are used to support the patient flow. Then, the demand side of a hospital consists of the many patients with different pathologies that enter the hospital according to their own time pattern, while the supply side consists on the one hand of the available personnel (e.g., surgeons, nursing staff) and on the other hand of the material resources (e.g., operating rooms, beds). The objective of a hospital is to match its supply and demand side in the best possible way, resulting in a quick, reliable and 
efficient patient service. However, the uncertainty existing in the health care system will play a destructive role on the efficiency of the health service delivery.

In this study, a hospital is assumed to be a profit maximizer. From an organizational perspective, the hospital is assumed to possess a fixed number of different departments and a common surgical center. Each department has its own surgeon groups and its own ward with a certain number of beds accommodating the recovery of its patients. Each surgeon group is engaged in one specialized medical field covering a few similar pathologies, and usually contains a certain number of equally qualified surgeons, who are inter-replaceable when performing surgery on their patients. The common surgical center is assumed to comprise a certain number of identical operating rooms (ORs), which are shared by all surgeon groups for performing surgeries. While hospital beds are allocated to each ward in a fixed way, operating rooms are assigned to surgeon groups in a time-phased manner. Clearly, operating rooms are the time-shared leading resources, while beds are the following resources. In the context of this research, emergency patients are assumed to be taken care of by the emergency department with the reserved capacity, and thus are left out of the planning process. The involved elective inpatients are classified into different patient groups according to an iso-process grouping procedure [6], and each patient group is characterized by a series of observed parameters: i.e., the average reward of the treatment, the specialized surgeon group, the expected surgery duration and the expected LOS with their probability distributions. Clearly, one surgeon group may perform surgeries on several different patient groups, but one patient group can only be treated by one surgeon group.

Figure 1 shows an example of the general hospital configuration, which clearly illustrates the relationship amongst wards, surgeon groups (S1 to S4) and patient groups (P1 to P9): e.g., surgeon group S1 takes care of patient groups P1 and P2, which will normally get recovery beds 
in Ward 1, and all patient groups P6 to P9, which are taken care of by surgeon groups S3 and S4 respectively, will normally end up in Ward 2.

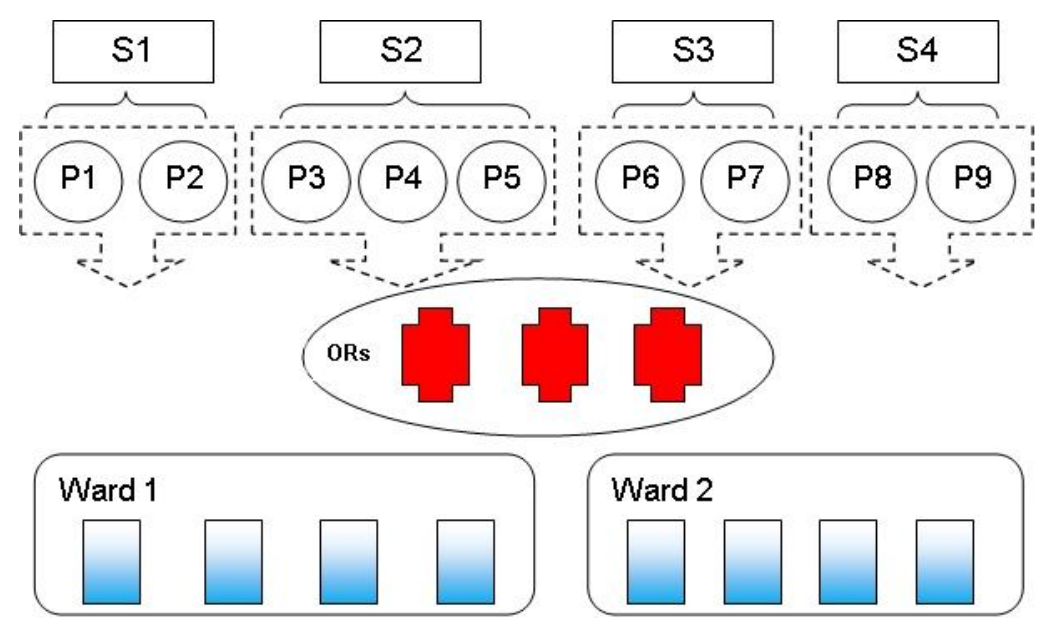

Figure 1: Hospital configuration (S: surgeon group, P: patient group)

Generally speaking, given the predictions of the number of patients that will enter a hospital with a certain pathology, given the expected financial reward, the expected operating time and the expected LOS per patient group and given the knowledge to which surgeon group and to which ward a patient with a certain pathology will be assigned, the basic hospital case mix and capacity planning problem consists of determining how operating rooms are assigned to surgeon groups, how many beds are allocated to each ward and how many patients from each pathology group can be taken care of at the hospital over the course of one year in view of maximizing the overall contribution. As a result, an optimal case mix pattern generating the maximum profits is sought, accompanied by a corresponding resource allocation scheme.

In previous research [11], the basic planning model generates the optimal case mix decision within a deterministic system, through matching the given resource capacity and various patient demands optimally from the perspective of resource efficiency. However, the uncertainty in the health care system, both from the arrival process and from the treatment process, not only upsets the resource utilization $[14,15,16]$, but further impacts the patient service level $[18,19,20,21]$. 
Therefore, in order to make sound decisions on case mix and resource allocation, it becomes of necessity to incorporate the variability into the capacity planning process.

Additionally, there are some other reasons influencing the medium-term decision on the resource allocation. First, according to our contacts with a number of well-reputed Belgian hospitals, we know that typically every six months (sometimes a year) a reallocation of capacity is performed based on proven capacity use in the previous six months (or a year). As such, the allocation of resources is based on historical 'rights' rather than on real data on the demand for and use of resources. Apparently, the current practice fails to provide a sound decision on the allocation of resources if a number of changes have taken place in the hospital during the period of six months, such as, the treatment of a certain pathology is changed (e.g., a different surgery procedure for a hip replacement results in a reduction of the average LOS from 9 to 7 days). The second possible reason considered is the time varying demand over the months, e.g., the seasonality in epidemics. Clearly, the patient arrivals in one department may vary periodically, while another department may have a countercyclical patient demand pattern. Then, for the hospital efficiency as a whole, it is helpful to allow borrowing beds from an idle department to a busy one or to set 'swing' beds that can be shared by clinical units that have countercyclical demand patterns [17].

\section{The multilevel integrative approach}

In this section, a multilevel model-based integrative approach is built to solve the hospital case mix and capacity planning problem. The solution methodology is based on operational research methods, such as mathematical programming and discrete-event simulation. It consists of three phases, each of which has its own emphasis and function and has a corresponding mathematical modeling. In the following, the methodology framework is formulated and explained clearly, and then each phase is elaborated in more detail. 


\subsection{Methodology framework}

The studied case mix and capacity planning problem has two distinct objectives: (1) maximizing the hospital resource efficiency and (2) improving the patient service level. Since each target has its own perspective and characteristics, it seems to be necessary to introduce a combined solution approach to achieve both objectives. Actually, our proposed solution approach comprises both a deterministic analysis and a stochastic analysis. The deterministic analysis allows to determine the optimal patient mix to be served, but it is unable to take the impact of variability into account. The stochastic analysis may reflect the influence of variability and explain the trade-offs between resource efficiency and service level for a given case mix, but does not give an optimal case mix. Clearly, both analyses are complementary in nature, and both are indispensable for studying the planning problem at hand in order to fulfill the two objectives.

Specifically, the proposed solution methodology includes three stages: i.e., the case mix planning phase, the master surgery scheduling (MSS) phase and the operational performance evaluation phase. The case mix planning phase involves the long-term decision making on patient volumes and capacity requirements of each specialty within a deterministic system, while the MSS phase considers the medium-term decision on resource allocation, i.e., a cyclic master surgery schedule, and takes into account the variability and its effect on resource utilizations, e.g., the expected bed occupancy of each ward. The performance evaluation phase is applied to assess and analyze the operational performance of a given case mix and capacity decision within a stochastic system.

Figure 2 displays the basic methodology framework. Clearly, there exists a loop among the three stages. Firstly, at the case mix planning phase, the optimal case mix pattern is sought, as well as the accompanying capacity allocation scheme is produced. Then, the solved case mix decision is communicated to the MSS phase, in which a 'balanced' master surgery schedule is generated 
with an improved resource utilization. Afterwards, the obtained case mix and capacity decision is translated into the operational performance phase, in which the performance evaluation is carried out in order to advance the health service level and to give valuable feedback to the upper stages. Thus, the three stages are interacting and are combined in an iterative way to form a multilevel integrative approach to the hospital planning problem with multiple objectives.

\section{Case mix planning phase}

Aim: maximizing the resource efficiency

Decision: case mix \& capacity allocation

Method: integer linear programming

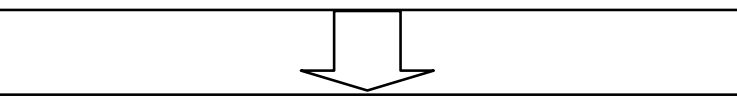

Master surgery scheduling phase

Aim: improving the resource utilization

Decision: cyclic master surgery schedule

Method: mixed integer programming

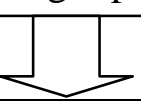

Performance evaluation phase

Aim: advancing the health service level

Decision: effective operations policies

Method: discrete-event simulation
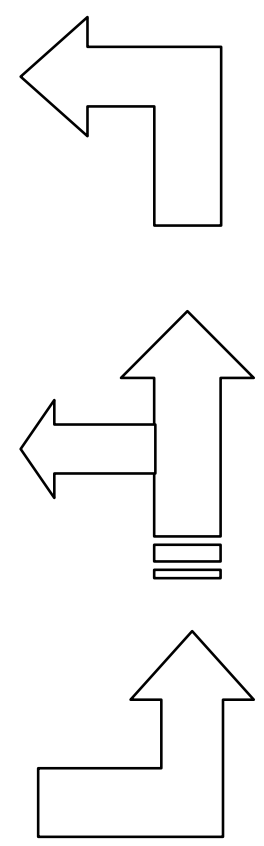

Interaction \& feedback Capacity:

Adapting the bed capacity
Variability:

Altering the probability distribution of the LOS, etc.

Figure 2: Methodology framework

At the case mix planning phase, the hospital is considered as a deterministic system, in which the patient treatment is following a standard care process for each patient group. The objective is to maximize the resource efficiency, so the hospital aims at choosing the optimal patient case mix that will bring the maximum overall financial contribution under the given resource capacity. In order to achieve this objective, a mathematical (integer linear) programming model is built to determine the optimal case mix pattern as well as the corresponding capacity allocation scheme. 
Then, the solved optimal case mix pattern is communicated as a constraint to the lower levels to make further decisions (e.g., the balanced master surgery schedule).

At the MSS phase, the variable resource requirement of each patient group is taken into account, which may impact the resource utilization, for example, the varied surgery duration may result in overtime in operating rooms, the variable LOS may result in bed shortages in a ward, and so on. Clearly, the variable LOS will impact the daily bed requirement of each ward, i.e., the expected bed demand may exceed the allocated bed capacity, which thus causes bed shortages. As a result, the bed shortage will force the scheduled surgeries to be cancelled or the operated patients to be misplaced, which lowers the patient service level. Hence, the objective at this level is to improve the resource utilization, i.e., minimizing the total expected bed shortage (TEBS) through leveling the daily bed occupancy of each ward. For this purpose, a mixed integer programming model is proposed to build a 'balanced' master surgery schedule with leveled resulting bed occupancy at each ward. Then, the 'balanced' master surgery schedule together with the optimal case mix will be evaluated under the variability condition at the operational level.

At the performance evaluation phase, the variability is introduced explicitly, such as the random patient arrivals and the stochastic resource requirements of each patient. For the purpose of the performance evaluation, a discrete-event simulation model is developed. The simulation analysis is applied to evaluate the operational performance of the provided case mix and capacity decision, and to analyze the suggested operations policies in order to improve the health care service level. The effective operations policies include adapting the bed capacity appropriately and altering the probability distribution of the LOS, both of which are helpful to advance the patient service level. Finally, the valuable effects of the operations policies are fed back to the upper stages to enhance the trade-offs between resource efficiency and service level. 


\subsection{Case mix planning}

At this level, a hospital is considered as a deterministic production system, in which each patient group represents a standard product type with a well-defined resource requirement structure. The patient treatment is following a standard procedure for each patient group. Then, patients within a same group will occupy the same constellation of resources, such as equal operating room time and equal bed days, and contribute the same reward to the hospital.

The determination of how many patients from each pathology group can be treated at a hospital over the course of one year is called the case mix planning problem. Aiming at maximizing the resource efficiency, the case mix planning problem will determine an optimal case mix pattern that can bring the maximum overall financial contribution, accompanied by a capacity allocation scheme over the whole hospital. To allocate the time-shared operating rooms to various surgeon groups, an OR block is defined as the smallest time unit for which an operating room can be assigned to a surgeon group to perform surgeries exclusively. The block length is usually set as eight hours, but this block length can easily be changed into other units (e.g., four hours). Here, it should be mentioned that the special surgery cases with a planned duration that is longer than the block length are excluded from this discussion. In addition, the case mix pattern is determined periodically with a cycle length of one week or a multiple of weeks.

In order to model the case mix planning problem mathematically, the required notation is defined as follows and remains valid for the rest of the paper. The needed sets and indices are:

$W$ : set of wards with index $w$;

$S$ : set of surgeon groups with index $s$;

$P$ : set of patient groups with index $p$;

$D$ : set of days in a cycle with index $d$; 
$A$ : set of active days in a cycle with index $a$;

$S_{w}$ : subset of surgeon groups $S$ whose patients are moved in ward $w$ after surgery is performed;

$P_{w}$ : subset of patient groups $P$ of which the patients occupy the beds of ward $w$;

$P_{S}$ : subset of patient groups $P$ of which the patients are operated on by surgeon group $s$;

$A_{p d}$ : subset of active days $A$ for which it applies that patients of group $p$ still stay in hospital on day $d$ if they underwent surgery on that day. (Remark: for the patient group $p$ with a mean

LOS being longer than the cycle length, an active day $a$ may be counted multiple times.)

The coefficients and the right hand side values are:

$r_{p}:$ the profit generated by treating a patient of patient group $p$;

$d u r_{p}$ : the surgery duration time of operating on a patient of patient group $p$;

$L B_{p}$ : the lower bound on the number of patients of group $p$ that can be treated per cycle;

$U B_{p}$ : the upper bound on the number of patients of group $p$ that can be treated per cycle;

$B E D S$ : the total number of available beds (assumed to be the existing bed capacity);

BLOCKS: the total number of OR blocks per day (assumed to be the daily block capacity);

LENGTH: the operating room block length (assumed to be 480 minutes).

In addition, the decision variables include:

$x_{p a}$ : the number of patients of patient group $p$ that receive surgery on day $a$;

$y_{w}$ : the number of beds assigned to ward $w$;

$z_{s a}:$ the number of OR blocks assigned to surgeon group $s$ on day $a$.

Then, the hospital case mix planning problem is formulated as an integer linear programming (ILP) model as below:

$$
\max \sum_{p \in P} r_{p} \sum_{a \in A} x_{p a}
$$




$$
\begin{array}{ll}
\text { s.t. } & \sum_{w \in W} y_{w} \leq B E D S, \\
& \sum_{p \in P_{w}} \sum_{a \in A_{p d}} x_{p a} \leq y_{w}, \quad \forall w \in W, d \in D, \\
& \sum_{s \in S} z_{s a} \leq B L O C K S, \quad \forall a \in A, \\
& \sum_{p \in P_{s}} d u r_{p} x_{p a} \leq z_{s a} L E N G T H, \quad \forall s \in S, a \in A, \\
& L B_{p} \leq \sum_{a \in A} x_{p a} \leq U B_{p}, \quad \forall p \in P, \\
& x_{p a}, y_{w}, z_{s a} \in \mathbb{Z}^{+}, \quad \forall w \in W, s \in S, p \in P, a \in A .
\end{array}
$$

The objective function (1) maximizes the total financial contribution. Constraint (2) indicates the allocation of bed capacity. Constraint set (3) denotes that the daily bed occupancy of each ward cannot exceed its allocated capacity. Constraint set (4) reflects the time-phased allocation of OR blocks. Constraint set (5) indicates that the total surgery time for each surgeon group on an active day cannot exceed the assigned block length. Constraints (6) denote the admission volume bound of each patient group and constraints (7) reflect the integer property of the decision variables.

Generally, the above formulation results in a huge integer program even for a hospital of regular size. For instance, for a hospital with 25 departments, if each department possesses three surgeon groups, each of which treats three patient groups on average, and if the cycle length is assumed to be one week, the ILP modeling will contain 1525 integer variables. Basically, no commercial ILP software (e.g., ILOG CPLEX) can solve an integer problem of this dimension effectively. In previous research [11], numerical experiments have shown that CPLEX fails to give an optimal or a feasible solution efficiently even for a hospital with five departments, but it is fortunate that an efficient branch-and-price approach has been developed to cope with this huge ILP model. 


\subsection{Master surgery scheduling}

At the case mix planning phase, besides an optimal case mix pattern, the corresponding resource capacity requirement of each specialty is also determined. However, the capacity allocation plan is a rough decision, since on the one hand it does not consider the variability and its impact on resource utilization and on the other hand it is a long-term decision, which neglects the mediumterm factors, such as the seasonality. As mentioned before, the variability will affect the resource utilization, and further impact the patient service. Therefore, it becomes important to incorporate the variability at the master surgery scheduling phase to determine the refined resource allocation at the medium-term level (e.g., the balanced cyclic master surgery schedule) from the perspective of the improved resource utilization.

As discussed above, both the cyclic master surgery schedule and the variable LOS of patients influence the daily bed occupancy of each ward. The variable LOS may introduce bed shortages, which thus lowers the patient service level. Hence, it is meaningful to reduce the total expected bed shortage as best as possible. In order to minimize the total expected bed shortage at the MSS phase, one attempts to take the variability of the LOS into account and to build a balanced master surgery schedule with a leveled resulting bed occupancy at each ward. At this phase, the concept of the expected bed shortage (EBS) is introduced to reflect the daily bed occupancy at each ward, and the probability distribution of the LOS of each patient group is applied to calculate the EBSs. Basically, the rough capacity decision at the case mix planning level may result in an unbalanced bed distribution (i.e., the possible spare beds are assigned to a few wards, which may cause a bed redundancy in some wards and a bed deficit in others) and the uneven peaks and troughs of bed occupancy at each ward. Then, a balanced master surgery schedule is built through reallocating the bed capacity and reassigning the OR blocks to level the bed occupancy of each ward. 
In order to build a balanced master surgery schedule with a leveled resulting bed occupancy, a mathematical (mixed integer) programming model that aims at minimizing the total expected bed shortage (TEBS) is proposed as below:

$$
\begin{aligned}
& \min \sum_{w \in W} T E B S_{w} \\
\text { s.t. } & \sum_{w \in W} y_{w} \leq B E D S, \\
& \sum_{s \in S} z_{s a} \leq B L O C K S, \quad \forall a \in A, \\
& \sum_{p \in P_{s}} d u r_{p} x_{p a} \leq z_{s a} L E N G T H, \quad \forall s \in S, a \in A, \\
& \sum_{a \in A} x_{p a}=T H R_{p}, \quad \forall p \in P, \\
& x_{p a}, y_{w}, z_{s a} \in \mathbb{Z}^{+}, \quad \forall w \in W, s \in S, p \in P, a \in A .
\end{aligned}
$$

In this model, $T E B S_{w}$ represents the total expected bed shortage of ward $w$, while $T H R_{p}$ denotes the patient throughput of pathology group $p$, which is received from the case mix planning level. This model seems to be similar with the one derived in the literature [23], but there are two main differences. First, the basic driving force to construct a master surgery schedule in this model is the case mix variable $x_{p a}$ instead of the block assignment variable $z_{s a}$. Thereby, in this case each surgeon group can perform a few different surgery types in one assigned OR block. Second, only one ward is considered in [23], whereas our proposed modeling involves multiple wards. Hence, this model is an improved extension to the one presented in [23]. However, similarly as pointed out in [23], the total expected bed shortage $T E B S_{w}$ of each ward cannot easily be expressed in terms of the decision variables $x_{p a}, y_{w}, z_{s a}$ due to the complicated combinations of the stochastic variables of the bed occupancy volume, which makes the above model intractable. 
To be able to solve the MSS problem effectively, we present an efficient approximation to the objective function (8), instead of calculating the complicated $T E B S_{w}$ directly. In the following, a simple version of the mixed integer programming (MIP) model is addressed to approximate the minimization of the total expected bed shortage over all wards. Moreover, in order to generate the master surgery schedule with more balanced bed occupancy levels, the OR blocks are relaxed to be more flexible (e.g., one 8-hour OR session is divided into two 4-hour sessions, or the block length is changed from 8 hours to 4 or 6 hours, but the total capacity of each OR-day is limited to at most 10 hours, etc). Then, a linear approximation MIP model is given below:

$$
\begin{aligned}
\min & \sum_{w \in W}\left(\alpha_{w} \sum_{d \in D} \delta_{w d}^{-}+\beta_{w} \delta_{w}^{+}+\gamma_{w} \sigma_{w}^{2}\right) \\
\text { s.t. } & \sum_{w \in W} y_{w} \leq B E D S, \\
& \mu_{w d}=\sum_{p \in P_{w}} \sum_{a \in A} x_{p a} \sum_{k \geq 0} \operatorname{prob}_{p, k T+d-a}, \\
& \sigma_{w d}^{2}=\sum_{p \in P_{w}} \sum_{a \in A} x_{p a} \sum_{k \geq 0} \text { prob }_{p, k T+d-a}\left(1-\text { prob }_{p, k T+d-a}\right), \\
& -\delta_{w d}^{-} \leq y_{w}-\mu_{w d} \leq \delta_{w d}^{+} \leq \delta_{w}^{+}, \quad \forall w \in W, d \in D, \\
& \sigma_{w d}^{2} \leq \sigma_{w}^{2}, \quad \forall w \in W, d \in D, \\
& \sum_{s \in S} \sum_{k \in K} z_{s a r k} L e n g t h_{k} \leq O R d a y_{a r}, \quad \forall a \in A, r \in R, \\
& \sum_{p \in P_{s}} d u r_{p} x_{p a} \leq \sum_{r \in R} \sum_{k \in K} z_{s a r k} L e n g t h_{k}, \quad \forall s \in S, a \in A, \\
& \sum_{a \in A} x_{p a}=T H R_{p}, \quad \forall p \in P, \\
& x_{p a}, y_{w} \in \mathbb{Z}^{+}, z_{s a r k} \in\{0,1\}, \delta_{w d}^{ \pm} \geq 0, \quad \forall w, s, p, a, d, r, k .
\end{aligned}
$$


The above model is applied to produce a balanced cyclic master surgery schedule with a leveled resulting bed occupancy. Here, constraints (11) and (12) respectively express the mean value and the variance of the bed requirement of ward $w$ on day $d$, in which $\operatorname{prob}_{p, l}$ denotes the probability that a patient of group $p$ is still an inpatient $l$ days after being operated on. In constraints (13), the negative deviation $\delta_{w d}^{-}$indicates the bed deficit and the positive deviation $\delta_{w d}^{+}$represents the bed surplus of ward $w$ on day $d$, while $\delta_{w}^{+}$denotes the maximum daily spare bed volume of ward $w$. Constraints (14) define the largest variance of the daily bed occupancy of each ward. In addition, constraints (15 - 16) are about the OR blocks, which are the variants of constraints (4) and (5) except that each operating room is considered individually and the blocks are differentiated into distinct lengths. $R$ denotes the set of rooms with an index $r$, and $K$ denotes the set of block types

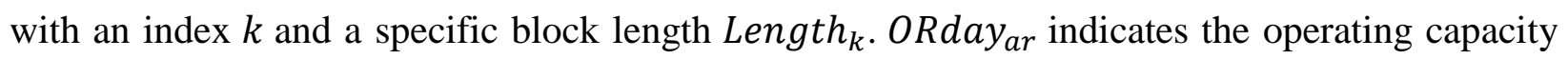
of room $r$ on day $a$, and the binary decision variable $z_{\text {sark }}$ implies whether the surgeon group $s$ obtains a block of type $k$ in room $r$ on day $a$. Constraints (15) ensure that for each OR-day the assigned surgery blocks cannot exceed its capacity, while constraints (16) guarantee that every surgeon group acquires enough OR blocks to perform its surgeries. The objective function (10) contains three weighted parts for each ward: i.e., the total bed deficit $\sum_{d \in D} \delta_{w d}^{-}$, the maximum daily spare bed volume $\delta_{w}^{+}$and the maximal variance of the bed occupancy $\sigma_{w}^{2}$.

Clearly, the MSS approach implements refining the resource capacity allocation. In addition, the MSS model is also applied to adjust the cyclic master surgery schedule at the medium-term level, according to the expected seasonal patient admission in place of the average target throughput of each patient group. The adaptation of the target patient throughput is helpful to reduce the patient waiting list. However, the revision should satisfy the condition that the given resource capacity is sufficient for each specialty in the deterministic environment. 


\subsection{Operational performance evaluation}

At the performance evaluation level, a discrete-event simulation model is developed to reflect the dynamic property of the health care system. Then, the variability within the health care system, either from the patient arrival process or from the patient treatment process, might be included explicitly. The simulation model is applied to evaluate the operational performance of the case mix and capacity decision that is obtained at the upper stages under the uncertainty condition. Moreover, in order to advance the trade-offs between resource efficiency and patient service, two effective operational policies are introduced, which are respectively adapting the bed capacity appropriately and altering the distribution of the variable LOS. The policies are analyzed through simulation experiments and the acquired valuable effects are fed back to the upper stages.

The discrete-event simulation model is built through using the user-friendly simulation software Arena version 11.0 [28]. It is elaborated in the previous research [21] and applied in this research to implement the performance evaluation. More specifically, a hospital is modeled as a stochastic production system, in which the random patient arrivals and the stochastic resource requirements of each patient are achieved by adopting probability distribution functions. Patient arrivals are created randomly and enter into each department. According to their pathology type, patients are classified into different groups and are put on the corresponding waiting lists. The master surgery schedule that is solved at the MSS stage determines on each day how many and which patients of every (first in first out) waiting queue are admitted and go into the surgical center to be operated on by the proper surgeon groups. Afterwards, patients will move to a proper ward to get recovery. If there exist bed shortages in the proper ward, the operated patient will be misplaced in a second suitable ward to recover temporarily. However, once a free bed is available in the proper ward, the misplaced patient will be transferred back from the wrong ward to the correct one. The LOS 
of each patient follows a certain probability distribution. When patients get recovered, they will leave the hospital in the end. Thereby, a simulation modeling of a hospital is portrayed from the perspective of the patient flow.

Ma and Demeulemeester [21] present a simulation modeling of a hospital with two departments. From the unit perspective, each department has its own patient arrival generators, which produce the patient arrivals of nine different pathology types randomly, as well as its corresponding three surgeon groups. Patients will wait for the admission and the treatment on their respective waiting lists. The surgical center has two operating rooms, which are shared and used by all six surgeon groups to perform surgeries on the total 18 patient groups, and the time-phased allocation of ORs is based on the obtained master surgery schedule. In addition, each department has an associated ward with a fixed number of beds to accommodate its patients for recovery. The number of beds at each ward is dependent on the planned resource allocation scheme. Besides, there exist a few temporary beds in the ward area, which will be used when no free bed is available at both wards. Clearly, the built simulation model by Arena is easily extendible and adaptable, so it is feasible to be applied in this research to implement the performance evaluation.

In order to be able to conduct the simulation experiments, the built simulation model needs to be concretized. The required data, such as the patient arrival rate of each pathology type, the cyclic master surgery schedule and the bed capacity allocation to each ward, as well as the expected surgery duration and the probability distribution of the variable LOS of each patient group, need to be specified in the simulation model. In addition, as the solved case mix and capacity decision plan is effective for six months to one year, the replication length is set as 365 days, in which the warm-up period is fixed as 60 days, and the number of replications is set as 50 to achieve reliable results in the simulation experiment. 
In order to guarantee the target patient throughput and thus the maximum financial contribution, the planned surgery will not be canceled when there exist bed shortages in the destined ward, but we adopt a policy that the operated patient will be misplaced in a second suitable ward to recover temporarily. Therefore, not the surgery cancellation but the patient misplacement is defined as the main performance indicator reflecting the patient service level, whereas the bed occupancy rate is another main performance indicator reflecting the resource utilization. The weekly volume of patient arrivals of every pathology is in accordance with the solved case mix pattern to ensure sufficient patient admissions. In addition, different from the variable LOS, the surgery length of each patient is assumed to be deterministic in the simulation model and is set as the mean value of its corresponding patient group for two reasons. First, the variable surgery duration does not affect the planned patient admission and the resulting bed occupancy, but only incurs the underor over-utilization of operating rooms. Second, the ORs' overtime to some extent is common and allowed in most Belgian hospitals. Hence, the variable surgery duration is not considered and the ORs utilization is not involved in this research.

The first objective of the performance evaluation phase is to assess and compare the operational performance of the produced various optimal case mix and capacity decisions through simulation experiments. Moreover, to advance the patient service level, two effective operational polices are proposed in this phase, which are analyzed through simulation experiments. Then, the obtained valuable information is fed back to the upper stages to further affect the case mix and capacity decision and to further influence the operational performance. The iteration is helpful to enhance the trade-offs between resource efficiency and service level, which is the second objective of this phase. The suggested two policies are respectively proposed from the perspective of capacity and of variability. 
The first efficient policy is to adapt the bed capacity appropriately. Because of the seasonality of patient arrivals, the bed requirement in some departments may be changed seasonally. That is, for one department there may exist a few spare beds at its ward during the low season, while the bed deficit may happen in a high season. However, for another department with countercyclical patient arrivals, there may appear a complementary bed requirement pattern. Then, for these two departments, if it is allowed to borrow/lend the spare beds from an idle department to a busy one temporarily, it will not only improve the bed utilization but also reduce the patient misplacement. If the simulation analysis shows that there exist bed shortages as a whole in the hospital in a busy period, we cope with this situation by staffing the stand-by beds during peak times or setting the 'swing' beds shared by the departments with countercyclical demand patterns at the operational level. Furthermore, the bed deficit/surplus status will be fed back to the tactical level to adapt the bed capacity appropriately, i.e., expanding or reducing the total bed capacity.

The second policy is to alter the probability distribution of the variable LOS properly. According to previous research [29], the patient delay and the bed utilization are tightly related with the variability within the healthcare system, which inspires us to examine the effect of the variability on the operational performance in this context. As mentioned before, a modified treatment of a pathology may cause the variable LOS to be changed, for example, the mean LOS is decreased and its probability distribution is varied as well. Undoubtedly, shortening the mean LOS of some pathologies will reduce both the bed occupancy and the patient misplacement as a whole, but the comparison seems to be unfair. Hence, we adopt a policy of changing the probability distribution but keeping the mean value of the variable LOS, which is called curtailing the distribution of the LOS (e.g., the distribution of a LOS with an interval between 3 and 11 days and an average of 7 days is changed to one with an interval between 5 and 9 days and an average of 7 days). The 
policy of curtailing the distribution of the LOS not only influences the operational performance but also affects the decision of the cyclic master surgery schedule at the MSS phase. Besides, the shortened average LOS will also affect the case mix and capacity planning. Therefore, adjusting the variable LOS of patients can be fed back to the upper stages to further influence the case mix and capacity decision.

\section{$4 \quad$ An application}

In this section, a small example is applied to demonstrate the efficiency and the effectiveness of the built integrated solution methodology to the hospital case mix and capacity planning problem. To implement the illustration, we built a small-sized simulated hospital following the general organizational structure and the basic operating principle of most Belgian hospitals. So, the small numerical example is representative and can easily be extended and adapted to the real situation. In the solution process, the case mix planning model is solved optimally by using the developed branch-and-price algorithm, and the MSS model is solved with the commercial ILP solver ILOG CPLEX. Then, the created discrete-event simulation model by Arena is launched to perform the simulation analysis, and two groups of simulation experiments are conducted to interpret the two proposed operational policies.

The fictitious hospital is assumed to have two departments (Dept. A and Dept. B) and a common surgical center with two time-shared operating rooms. Each department has three surgeon groups and each surgeon group can perform surgeries for three patient groups. Two operating rooms are open for eight hours on each working day, which are assigned to the total six surgeon groups based on the obtained resource allocation scheme. In addition, the random patient arrival of each pathology follows a certain non-stationary Poisson process. The expected surgery length of each pathology type is generated randomly from a uniform distribution between one and four hours. 
The variable LOS of each patient group is described by a discrete probability distribution. For example, a variable LOS with an average of $l$ days has the probability distribution as follows: $p(l-1)=0.3, p(l)=0.45, p(l+1)=0.2$ and $p(l+2)=0.05$.

The first experiment is to test the policy of adapting the bed capacity appropriately. According to the result of numerical experiments for the given hospital, when the total bed capacity reaches 38 beds, the overall financial contribution arrives at the maximum value in the deterministic phase. At that time, operating rooms become a bottleneck resource. A bed expansion cannot increase the total profits but will influence the operational performance of the hospital under uncertainty. Thus, we devise a simulation experiment to evaluate and to compare the operational performance of three different bed capacity cases (i.e., 38 beds, 40 beds and 41 beds). The simulation results are displayed and compared in Table 1, in which the 'Base' scenario refers to the rough resource allocation scheme by solving the case mix model, while the '+MSS' scenario refers to the refined resource allocation by further solving the MSS model.

\begin{tabular}{|c|c|c|c|c|c|c|c|}
\hline \multirow{2}{*}{\multicolumn{2}{|c|}{$\begin{array}{l}\text { Exp.1: Adapting the bed } \\
\text { capacity appropriately }\end{array}$}} & \multicolumn{2}{|c|}{38 beds } & \multicolumn{2}{|c|}{40 beds } & \multicolumn{2}{|c|}{41 beds } \\
\hline & & Base & $+\mathrm{MSS}$ & Base & $+\mathrm{MSS}$ & Base & $+\mathrm{MSS}$ \\
\hline Patient & A's MBDs & 60.04 & 30.68 & 31.38 & 6.08 & 17.42 & 5.42 \\
\hline Misplacement & B's MBDs & 54.02 & 34.74 & 9.40 & 10.72 & 4.12 & 2.72 \\
\hline Bed Days & $\mathrm{AB}$ in $\mathrm{T}$ & 56.80 & 27.42 & 8.64 & 4.34 & 2.46 & 1.32 \\
\hline (MBDs) & Total & 114.06 & 65.42 & 40.78 & 16.80 & 21.54 & 8.14 \\
\hline & & 0.8566 & 0.8576 & 0.8581 & 0.7987 & 0.7894 & 0.7973 \\
\hline Occupancy & Ward A & 10.2792 & 10.2912 & 10.2966 & 10.3831 & 10.2620 & 10.3652 \\
\hline Rate \& & & 0.9028 & 0.9058 & 0.8494 & 0.8767 & 0.8384 & 0.8464 \\
\hline Number & Ward B & 23.4739 & 23.5505 & 23.7827 & 23.6704 & 23.4764 & 23.6979 \\
\hline
\end{tabular}

Table 1: Comparison results of adapting the total bed capacity 
The main performance indicators are compared in Table 1, comprising the patient misplacement bed days (MBDs) and the average bed occupancy of each ward. Here, 'AB in T' represents the total misplacement bed days of patient A (patients of Dept. A) and patient B (patients of Dept. B) on temporary beds, which are occupied as both wards have no free bed available. A(B)'s MBDs consist of the misplacement bed days of patient $\mathrm{A}(\mathrm{B})$ both in ward $\mathrm{B}(\mathrm{A})$ and on temporary beds. Besides, 'Total' reflects the total misplaced bed days of patients A and B. Clearly, for each case, the refined resource allocation scenario has much better operational performance than the rough resource allocation scenario on the whole, as both the ' $\mathrm{AB}$ in $\mathrm{T}$ ' and the total MBDs demonstrate a definite decrease in total. In the first and the third cases, both patient A's MBDs and patient B's MBDs have evident decreases, and the bed occupancy rate shows a little increment. However, in the second case, patient B's MBDs have some increment in the '+MSS' scenario and the average bed occupancy rates at two wards have an inverse change. This is because a spare bed of Ward B is reallocated to Ward A in the refined resource allocation plan. On the other hand, the quite high bed occupancy rate (more than $85 \%$ at both wards) in the case of 38 beds implies the necessity of expanding the total bed capacity. Clearly, when the total bed capacity reaches 41 beds, the bed occupancy rates of both wards decrease to lower than the baseline of $85 \%$. In general, as the bed capacity gets expansion, both the total patient misplacement and the average bed occupancy have evident improvements, which thus advances the trade-offs between the patient service level and the hospital resource efficiency.

The second experiment is to examine the efficiency of curtailing the distribution of the variable LOSs. To reflect the possible treatment improvement for some certain pathologies, it is assumed that the long-tailed LOS of some patient groups can get effectively controlled, which refers to the second policy that we introduced. In our numerical experiments, a variable LOS with an average 
of $l$ days and the probability distribution of $p(l-2)=0.1, p(l-1)=0.2, p(l)=0.45$, $p(l+1)=0.15, p(l+2)=0.05$ and $p(l+3)=0.05$ is changed to one with a distribution of $p(l-1)=0.3, p(l)=0.45, p(l+1)=0.2$ and $p(l+2)=0.05$.

Figure 3 exhibits the effect of curtailing the distribution of the variable LOSs via the simulation experiments. Here, the 'Base' scenario denotes the rough resource allocation without considering the variability, the 'Balanced' scenario refers to the refined resource allocation after taking into account the variability, the mixed ' $\mathrm{B} \& \mathrm{C}$ ' scenario relates the refined resource allocation with the curtailing information in the performance evaluation phase, while the 'Curtailed' scenario refers to the updated resource allocation after feeding back the curtailing information to the MSS stage. Apparently, the average bed occupancy rates remain constant, since the required overall bed days are unchanged. However, the patient misplacement bed days show definite improvements when considering the variability in different levels, which clearly reveals that the higher the degree of variability, the worse the operational performance will be for the same utilization level.

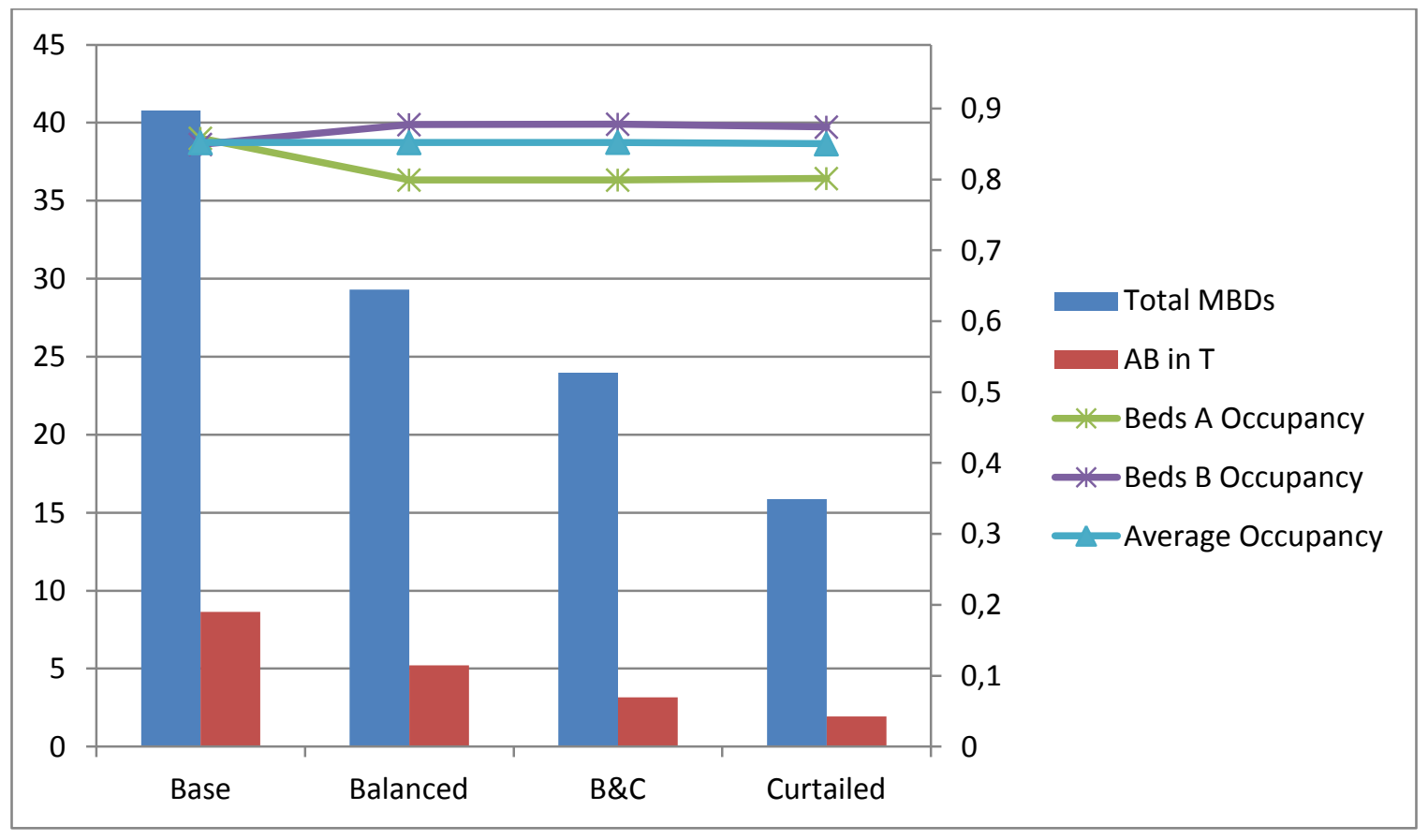

Figure 3: The effect of curtailing the distribution of the variable LOSs 
According to the above simulation results, it is clearly shown that the operational performance of the optimal case mix and capacity decision derived from the proposed methodology, such as the '+MSS' scenario or the 'Curtailed' scenario, presents visible improvements compared to the base case scenario derived from a deterministic approach. Therefore, the proposed integrative solution methodology is demonstrated to be effective to enhance the efficient trade-offs between hospital efficiency and patient service.

\section{Conclusions and future research}

This paper presents a multilevel integrative solution approach to a hospital case mix and capacity planning problem on the basis of mathematical programming and simulation analysis. The case mix and capacity planning problem aims at advancing both the resource efficiency and the health service level. In order to maximize the hospital resource efficiency, an optimization (ILP) model is developed to solve the optimal case mix pattern and the associated resource allocation scheme in a deterministic manner. However, the existing variability within a health care system not only affects the resource utilization but further impacts the health service delivery. Hence, we take the variability into account to absorb its effects and to improve the time-phased resource allocation. Moreover, a discrete-event simulation model is built to evaluate the operational performance and to analyze how to enhance the trade-offs between efficiency and service.

A numerical example is applied to illustrate the efficiency of the proposed solution methodology. Then, it would be more valuable to apply this methodology to a real case situation in a Belgian hospital, in which the seasonality of the patient arrivals can be included instead of considering an identical patient demand over the whole year in the numerical example. The time-varying patient demands of some pathologies reflect the real situation in a hospital, but may challenge the timephased resource allocation of the planning problem. To cope with this issue, we might adopt the 
flexible master surgery scheduling, i.e., generating the seasonal master surgery schedules, which is helpful to increase the resource utilization and to reduce the patient waiting list.

This paper works on a study of the case mix and capacity planning for elective inpatients, while emergency patients are excluded from the discussion since the case mix planning is infeasible for emergency patients because of their random arrival property. Actually, according to a prediction for the amount of emergencies, it is assumed that there are a certain number of reserved resource capacities exclusively utilized by the emergency patient in our healthcare setting. Nevertheless, it is feasible to incorporate the emergency admission at the resources planning level to improve the time-phased resource allocation, which may advance both the resource utilization and the patient service. Other possible extensions can be formed through including some other resources such as intensive care beds or by integrating the nursing staff planning and scheduling.

In addition, the studied case mix and capacity planning problem considers a whole hospital as the main unit of analysis. The hospital planning decides a tactical master plan for elective inpatients and the resource allocation over a whole hospital, and the performance evaluation is also carried out from a tactical perspective. However, the planning problem can be extended over a hospital network in a certain area, and the tactical case mix and capacity planning can be combined with the operational patient scheduling to form an efficient health care production framework.

\section{Acknowledgements}

The authors acknowledge the support given to this project by the Fonds voor Wetenschappelijk Onderzoek (FWO)-Vlaanderen, Belgium under contract number G.0456.08.

\section{References}

[1] RIZIV. Evolutie van de uitgaven voor geneeskundige verzorging. www.riziv.be; 2010.

[2] OECD. OECD health data: Statistics and indicators for 34 countries. www.oecd.org; 2010. 
[3] Carter M. Diagnosis: Mismanagement of resources. ORMS Today 2002; 29: 26-32.

[4] Vissers J. Aggregate hospital production and capacity planning. In: Vissers J, Beech R, editors. Health Operations Management: Patient flow logistics in health care, New York: Routledge; 2005, p. 116-45.

[5] Fetter RB, Shin Y, Freeman JL, Averill RF, Thompson JD. Case mix definition by diagnosisrelated groups. Medical Care 1980; 18: 1-53.

[6] Vissers, JMH, Bertrand JWM, de Vries G. A framework for production control in healthcare organizations. Production Planning \& Control 2001; 12: 591-604.

[7] Simon HA. The new science of management decision. New York: Harper \& Row; 1960.

[8] Blake JT, Carter MW. A goal programming approach to strategic resource allocation in acute care hospitals. European Journal of Operational Research 2002; 140: 541-61.

[9] Brandeau ML, Hopkins D. A patient mix model for hospital financial planning. Inquiry 1984; 21: $32-44$.

[10] Mulholland MW, Abrahamse P, Bahl V. Linear programming to optimize performance in a department of surgery. Journal of the American College of Surgeons 2005; 200: 861-8.

[11] Ma G, Beliën J, Demeulemeester E, Wang L. Solving the strategic case mix problem optimally by using branch-and-price algorithms. In: Proceedings of the 35th International Conference on Operational Research Applied to Health Services, Leuven, Belgium; 2009.

[12] Barnhart C, Johnson EL, Nemhauser GL, Savelsbergh MWP, Vance PH. Branch-and-price: Column generation for solving huge integer programs. Operations Research 1998; 46: 316-29.

[13] Utley M, Gallivan S, Jit M. How to take variability into account when planning the capacity for a new hospital unit. In: Vissers J, Beech R, editors. Health Operations Management: Patient flow logistics in health care, New York: Routledge; 2005, p. 146-61. 
[14] Adan I, Bekkers J, Dellaert N, Vissers J, Yu X. Patient mix optimisation and stochastic resource requirements: A case study in cardiothoracic surgery planning. Health Care Management Science 2009; 12: 129-41.

[15] Bagust A, Place M, Posnett JW. Dynamics of bed use in accommodating emergency admissions: Stochastic simulation model. British Medical Journal 1999; 319: 155-8.

[16] Gallivan S, Utley M, Treasure T, Valencia O. Booked inpatient admissions and hospital capacity: Mathematical modeling study. British Medical Journal 2002; 324: 280-2.

[17] Green LV. Capacity planning and management in hospitals. In: Brandeau ML, Sainfort F, Pierskalla WP, editors. Operations Research and Health Care: A handbook of methods and applications, Boston: Kluwer Academic Publishers; 2004, p. 15-41.

[18] Harper PR, Shahani AK. Modelling for the planning and management of bed capacities in hospitals. Journal of the Operational Research Society 2002; 53: 11-8.

[19] VanBerkel P, Blake J. A comprehensive simulation for wait time reduction and capacity planning applied in general surgery. Health Care Management Science 2007; 10: 373-85.

[20] Adan IJBF, Bekkers J, Dellaert NP, Jeunet J, Vissers JMH. Improving operational effectiveness of tactical master plans for emergency and elective patients under stochastic demand and capacitated resources. Eurandom Report (Ext. rep. 2009-052), Eindhoven; 2009.

[21] Ma G, Demeulemeester E. Assessing the performance of hospital capacity planning through simulation analysis. FBE Research Report KBI_1031, Katholieke Universiteit Leuven, Belgium; 2010.

[22] Beliën J, Demeulemeester E, Cardoen B. Visualizing the demand for various resources as a function of the master surgery schedule: A case study. Journal of Medical Systems 2006; 30: 343-50. 
[23] Beliën J, Demeulemeester E. Building cyclic master surgery schedules with leveled resulting bed occupancy. European Journal of Operational Research 2007; 176: 1185-204.

[24] van Oostrum JM, Van Houdenhoven M, Hurink JL, Hans EW, Wullink G, Kazemier G. A master surgical scheduling approach for cyclic scheduling in operating room departments. OR Spectrum 2008; 30: 355-74.

[25] Bretthauer KM, Côté MJ. A model for planning resource requirements in health care organizations. Decision Sciences 1998; 29: 243-70.

[26] Butler TW, Karwan KR, Sweigart JR, Reeves GR. An integrative model-based approach to hospital layout. IIE Transactions 1992; 24: 144-52.

[27] Oddoye JP, Jones DF, Tamiz M, Schmidt P. Combining simulation and goal programming for healthcare planning in a medical assessment unit. European Journal of Operational Research 2009; 193: 250-61.

[28] Kelton WD, Sadowski RP, Sturrock DT. Simulation with Arena. 4th ed. New York: McGraw Hall; 2008.

[29] Green L. Queueing analysis in healthcare. In: Hall RW, editor. Patient flow: Reducing delay in healthcare delivery, New York: Springer Science; 2006, p. 281-307. 